\title{
IAP Textbook of Vaccines: IAP Committee on Immunization (IAPCOI), 2011-13 (eds)
}

\author{
Published by Jaypee Brothers Medical Publishers (P) Ltd., ISBN 978-93-5090-947-8, First \\ Edition: 2014
}

\author{
A. K. Dutta
}

Received: 23 July 2014 / Accepted: 12 August 2014 /Published online: 29 August 2014

(C) Dr. K C Chaudhuri Foundation 2014

Vaccination is one of the most cost effective method of prevention of infectious diseases. Since the invention of Small Pox vaccine by Sir Edward Jenner, a large number of vaccines are available in the world which have prevented several million of deaths, disabilities and morbidities in children. Successful immunization practices have led to eradication of Small Pox from the world and in near future, Poliomyelitis would be a disease of the past. In developed countries of the world, many diseases are in control because of inclusion of the vaccines in the country program. Immunization program of a country depends on disease burden, availability of vaccines, feasibility of implementation and cost effectiveness. Based on the above issues, every country decides its own immunization schedule. However, there are several vaccines which are not included in the national schedule but are available in the country for private use. Indian Academy of Pediatrics has time to time given guidelines for all the members to use the additional vaccines which are to be used for children under their care. It has been observed in the past that there is always debate regarding various aspects of immunization practices and the continuing medical education on vaccination attracts most captive audiences. Most of the time it becomes difficult for the organizer as well the speaker to satisfy the delegates. The IAP text book of vaccines is a long awaited prized publication which shall serve as a reference book for every pediatrician and physician practicing immunization.

The book has been edited by eminent personalities in the field of vaccination and all contributors from India and abroad are experts in this field. The book includes excellent chapters from basics of vaccination to details of individual vaccines covering epidemiology, clinical presentations and vaccine administration. The chapter on history of vaccination is very interesting to read. The initial chapters e.g., immunological correlates of protection induced by vaccine, epidemiology in relation to vaccinology, general recommendations on immunization, scheduling of vaccine, mucosal immunology and vaccination are very informative.

The section 3 on new vaccines in development, contains important vaccines which are in the pipeline and latest and most recent developments in individual vaccine development has been nicely written by all the authors. It is very heartening to find one of the most important chapter on vaccine safety and adverse reaction to vaccination and what needs to be done in case of such an event including management in the community and media.

The book is complete in respect of its usefulness to all spheres of medical profession e.g., in government set up, as policy makers, for all pediatricians and physicians and also to the vaccine manufacturing industry.

The book is an excellent reference publication by Indian Academy of Pediatrics with very good editing, covering all aspects of vaccination. I am sure this book should find place in the library of each medical college, referral hospitals in the country and also in the desk of every pediatrician and physician practicing immunization.

\footnotetext{
A. K. Dutta $(\bowtie)$

Department of Pediatrics, SMSR, Sharda University,

Greater Noida, Uttar Pradesh 201306, India

e-mail: drdutta@gmail.com
} 